\title{
Near-Field Effects in Spatial Coherence of Thermal Sources
}

\author{
Rémi Carminati and Jean-Jacques Greffet \\ Laboratoire d'Energétique Moléculaire et Macroscopique, Combustion, Ecole Centrale Paris, \\ Centre National de la Recherche Scientifique, 92295 Châtenay-Malabry Cedex, France
}

(Received 16 October 1998)

\begin{abstract}
We present an exact calculation of the cross-spectral density tensor of the near field thermally emitted into free space by an opaque planar surface. The approach, based on fluctuational electrodynamics and the fluctuation-dissipation theorem, yields novel near-field correlation properties. We show that the spatial coherence length of the field close to the surface at a given wavelength $\lambda$ may be much smaller than the well-known $\lambda / 2$ of blackbody radiation. We also show that a long-range correlation may exist, when resonant surface waves, such as surface-plasmon or surface-phonon polaritons, are excited. These results should have important consequences in the study of coherence in thermal emission and in the modeling of nanometer scale radiative transfer. [S0031-9007(99)08515-4]
\end{abstract}

PACS numbers: 42.25.Kb, 05.40.-a, 71.36.+c, 78.20.Ci

Thermal emission of radiation from opaque materials is often regarded as an incoherent phenomenon. Indeed, thermal light is chiefly generated by the uncorrelated process of spontaneous emission. Nevertheless, since the development of optical coherence theory, it has been known that the field emitted by thermal sources may exhibit a certain degree of temporal and spatial coherence. For example, it was shown that light across a planar quasihomogeneous Lambertian source, at a given wavelength $\lambda$, is spatially correlated over a distance on the order of $\lambda / 2$ [1]. This result was found to be in agreement with known spatial correlation properties of free blackbody radiation [2].

In deriving this result, the nonradiating near-field part of the emitted light was ignored [1], because it plays no role in the far-field properties of emission from planar sources. Nevertheless, recent interest in microscale and nanoscale radiative transfers [3], together with the development of local-probe thermal microscopy [4] and the observation of coherent thermal emission from doped silicon and silicon carbide ( $\mathrm{SiC}$ ) gratings [5,6], has raised new challenges. In fact, all these topics have in common the substantial role of the nonradiating (evanescent) thermal fields.

In this Letter, we concentrate on the role of the nonradiating field in the coherence properties of thermal sources, with particular emphasis on the spatial coherence. We revisit this concept by studying the spatial correlation of the vector thermal field at close proximity of an emitting body. Our calculation is based on macroscopic fluctuational electrodynamics and the fluctuation-dissipation theorem $[7,8]$. Novel properties are obtained, showing that near-field effects may dramatically modify the spatial coherence of thermal sources.

Let us consider a homogeneous opaque material filling the half space $z<0$, in local thermodynamic equilibrium at a uniform temperature $T$. The electrodynamic properties of the material, assumed to be isotropic and nonmagnetic, are macroscopically described by its complex frequency-dependent dielectric constant $\epsilon(\omega)$. The thermal fluctuations of the current density at a point $\mathbf{r}=$ $(x, y, z)$ inside the body are described by a random process $\mathbf{j}(\mathbf{r}, t)$, which is stationary in time [7,8]. The field radiated into the half space $z>0$, i.e., the thermally emitted field, is itself a fluctuating quantity described by a timestationary random process $\mathbf{E}(\mathbf{r}, t)$. In this Letter, we focus on the spatial coherence of the emitted field at a given frequency $\omega$. The basic quantity of second-order coherence theory of vector fields in the space-frequency domain is the electric-field cross-spectral density tensor $W_{j k}$ defined by [9]

$$
\left\langle E_{j}\left(\mathbf{r}_{1}, \omega\right) E_{k}^{*}\left(\mathbf{r}_{2}, \omega^{\prime}\right)\right\rangle=W_{j k}\left(\mathbf{r}_{1}, \mathbf{r}_{2}, \omega\right) \delta\left(\omega-\omega^{\prime}\right),
$$

where $\mathbf{E}(\mathbf{r}, \omega)$ is the time-domain Fourier transform of the electric field $\mathbf{E}(\mathbf{r}, t)$ and the superscript $*$ denotes the complex conjugate. The brackets denote a statistical ensemble average. The tensor $W_{j k}$ is a measure of the spatial correlation of the electric field at a given frequency $\omega$ and at two different points $\mathbf{r}_{1}$ and $\mathbf{r}_{2}$. Note that the presence of the delta function in Eq. (1) is a consequence of the stationarity of the field in the time domain. Recently, calculation of the cross-spectral density tensor $W_{j k}$ was reported for an infinite blackbody surface in the far field or for a finite planar surface in the radiometric limit [10]. In this Letter, we address near-field effects - in some cases under resonant conditions - so that we do not use any approximation in our calculation. The only assumptions are the local thermal equilibrium of the body and the use of macroscopic electrodynamics. To proceed, we introduce the time-domain Fourier transform $\mathbf{j}(\mathbf{r}, \omega)$ of the random current density $\mathbf{j}(\mathbf{r}, t)$. The electric field at a given point $\mathbf{r}$ in the half space $z>0$ is given by

$$
\mathbf{E}(\mathbf{r}, \omega)=i \mu_{o} \omega \int_{V} \overleftrightarrow{\mathbf{G}}\left(\mathbf{r}, \mathbf{r}^{\prime}, \omega\right) \cdot \mathbf{j}\left(\mathbf{r}^{\prime}, \omega\right) d^{3} \mathbf{r}^{\prime},
$$

where the integration is performed on the volume $V$ of the body. $\overleftrightarrow{\mathbf{G}}\left(\mathbf{r}, \mathbf{r}^{\prime}, \omega\right)$ is the Green dyadic of the vector Helmholtz equation in the considered geometry, namely, a flat interface separating a semi-infinite homogeneous 
medium with dielectric constant $\epsilon(\omega)$ (medium $z<0$ ) from a vacuum (medium $z>0$ ) [11].

In order to calculate the cross-spectral density tensor $W_{j k}$, we need to know the spatial correlation function of the thermal current fluctuations in the frequency domain. It is given by the fluctuation-dissipation theorem [7,8]:

$$
\begin{aligned}
& \left\langle j_{m}(\mathbf{r}, \omega) j_{n}^{*}\left(\mathbf{r}^{\prime}, \omega^{\prime}\right)\right\rangle= \\
& \frac{\omega}{\pi} \epsilon_{o} \epsilon^{\prime \prime}(\omega) \Theta(\omega, T) \delta\left(\mathbf{r}-\mathbf{r}^{\prime}\right) \delta_{m n} \delta\left(\omega-\omega^{\prime}\right),
\end{aligned}
$$

where $\delta_{m n}$ is the Kronecker symbol, $\Theta(\omega, T)=\hbar \omega / 2+$ $\hbar \omega /[\exp (\hbar \omega / k T)-1]$ is the mean energy of the quantum harmonic oscillator in thermal equilibrium at temperature $T$, and $2 \pi \hbar$ is Planck's constant. $\epsilon^{\prime \prime}(\omega)$ is the imaginary part of the dielectric constant $\epsilon(\omega)$.

Inserting Eq. (2) into Eq. (1) and using Eq. (3), we obtain the following expression for the cross-spectral tensor of the electric field in the half space $z>0$ :

$$
\begin{aligned}
& W_{j k}\left(\mathbf{r}_{1}, \mathbf{r}_{2}, \omega\right)= \\
& \frac{\omega^{3}}{\pi} \mu_{o}^{2} \epsilon_{o} \epsilon^{\prime \prime} \Theta(\omega, T) \int_{V} G_{j m}\left(\mathbf{r}_{1}, \mathbf{r}^{\prime}, \omega\right) G_{k m}^{*}\left(\mathbf{r}_{2}, \mathbf{r}^{\prime}, \omega\right) d^{3} \mathbf{r}^{\prime} .
\end{aligned}
$$

The expression of the Green dyadic $\overleftrightarrow{\mathbf{G}}\left(\mathbf{r}, \mathbf{r}^{\prime}, \omega\right)$ connecting a source current inside the body (medium $z<0$ ) to the electric field in vacuum (medium $z>0$ ) is [11]

$$
\begin{aligned}
\overleftrightarrow{\mathbf{G}}\left(\mathbf{r}, \mathbf{r}^{\prime}, \omega\right) & = \\
\frac{i}{8 \pi^{2}} \int \frac{1}{\gamma_{2}}\left(\hat{s} t_{s} \hat{s}\right. & \left.+\hat{p}_{1} t_{p} \hat{p}_{2}\right) \exp \left[i \mathbf{K} \cdot\left(\mathbf{R}-\mathbf{R}^{\prime}\right)\right] \\
& \times \exp \left(i \gamma_{1} z-i \gamma_{2} z^{\prime}\right) d^{2} \mathbf{K},
\end{aligned}
$$

where $\mathbf{r}=(\mathbf{R}, z), \hat{s}=\hat{\mathbf{K}} \times \hat{z}, \hat{p}_{j}=\left(|\mathbf{K}| \hat{z}+\gamma_{j} \hat{\mathbf{K}}\right) / k_{j}$, the symbol ${ }^{\wedge}$ denoting a unit vector, $k_{1}=k=\omega / c$, $k_{2}=\sqrt{\epsilon} k$, and $\gamma_{j}=\left(k_{j}^{2}-\mathbf{K}^{2}\right)^{1 / 2}$, with the determination $\operatorname{Re}\left(\gamma_{j}\right)>0$ and $\operatorname{Im}\left(\gamma_{j}\right)>0$. $t_{s}(\mathbf{K})$ and $t_{p}(\mathbf{K})$ are the Fresnel transmission factors for $s$ and $p$ polarizations, respectively [11].

Equation (4), together with Eq. (5), gives an exact expression of the electric cross-spectral density tensor $W_{j k}$. This expression is valid for any position of the two observation points $\mathbf{r}_{1}$ and $\mathbf{r}_{2}$, especially at close proximity from the surface (near-field zone). Inserting (5) into (4), tedious but straightforward algebra allows us to express the elements $W_{j k}\left(\mathbf{r}_{1}, \mathbf{r}_{2}, \omega\right)$ of the cross-spectral tensor in terms of a single integral over the wave vector $|\mathbf{K}|$ which is evaluated numerically.

Let us first compare the spatial correlation of the field emitted by lossy glass and tungsten, the latter being a metal which does not exhibit surface-polariton resonances in the visible part of the spectrum. We plot in Fig. 1 the diagonal element $W_{x x}\left(\mathbf{r}_{1}, \mathbf{r}_{2}, \omega\right)$ of the cross-spectral density tensor, at a wavelength $\lambda=2 \pi / k=500 \mathrm{~nm}$ and normalized by its value at $\rho=0$. Note that this normalization amounts to plotting the component $\mu_{x x}\left(\mathbf{r}_{1}, \mathbf{r}_{2}, \omega\right)$ of the (tensor) spectral degree of spatial coherence [9]. At this wavelength, the dielectric constant of a lossy glass is $\epsilon=$
$2.25+0.001 i$ and that of tungsten is $\epsilon=4.35+18.05 i$. The calculation is performed in a plane $z=z_{0}$ above the surface of the emitting material. Both $\mathbf{r}_{1}$ and $\mathbf{r}_{2}$ are along the $x$ axis, and the result is plotted versus $\rho=\left|\mathbf{r}_{1}-\mathbf{r}_{2}\right|$. In the very near field $\left(z_{0}=0.01 \lambda\right)$, the curve corresponding to glass (solid line) drops to negligible values after $\rho=\lambda / 2$, showing that the correlation length of the $x$ component of the field is $\lambda / 2$. In fact, a semi-infinite medium of lossy glass is a good approximation of a planar blackbody radiator, and the solid curve in Fig. 1 strongly resembles the $\sin (k r) / k r$ shape of the cross-spectral density in the source plane of a Lambertian source, previously obtained in the scalar approximation [1]. Note that, although not shown here for brevity, we have observed this behavior for the three diagonal elements $W_{j j}$ of the cross-spectral density tensor. In comparison, the case of tungsten (dotted curve) is completely different. The correlation length is much smaller than $\lambda / 2$, on the order of $0.06 \lambda$. This distance is comparable to the skin depth $\delta=[k \operatorname{Im}(\sqrt{\epsilon})]^{-1}$ of tungsten at this wavelength. This is actually not surprising because $\delta$ is, in addition to the wavelength, the relevant length scale for the propagation of electromagnetic waves inside the material. Therefore, it is physically sound that the induced currents, and thus the field close to the surface, be correlated over a distance which is the smallest of $\delta$ and $\lambda$. This result, which, to our knowledge, was never pointed out before, has important consequences in the modeling of radiative transfer at small scales $[3,4]$. Moreover, it allows us to revisit the concept of emissivity at subwavelength scale, useful in the study of the radiative properties of rough surfaces [12]. Indeed, this macroscopic concept can be defined on a length scale larger than the field correlation length.

Finally, we show that this subwavelength correlation length is a pure near-field effect, due to nonradiative evanescent fields. At a distance $z_{0}=0.1 \lambda$, we see that the correlation length for tungsten (dashed curve in Fig. 1) is much larger (on the order of $0.4 \lambda$ ) than that obtained with

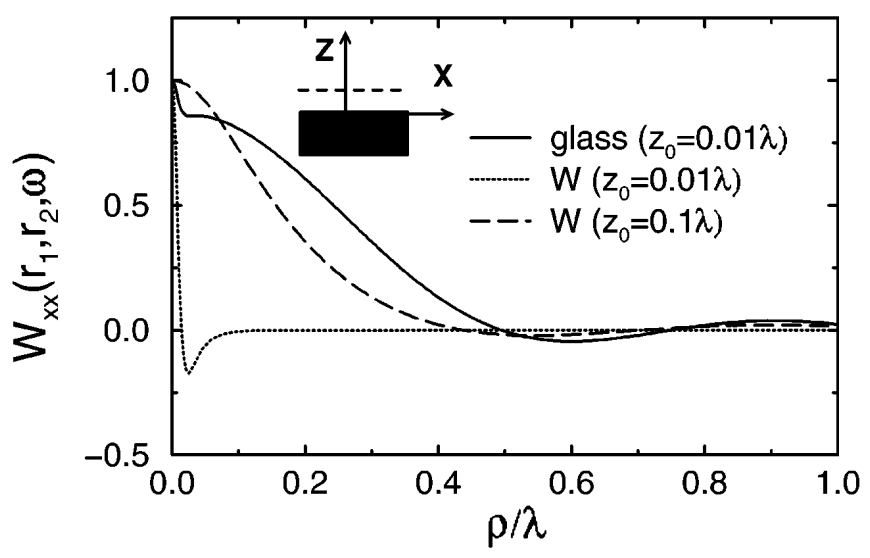

FIG. 1. $W_{x x}\left(\mathbf{r}_{1}, \mathbf{r}_{2}, \omega\right)$ in the plane $z=z_{0}$ versus $\rho=\mid \mathbf{r}_{1}-$ $\mathbf{r}_{2}$. $\quad \mathbf{r}_{1}$ and $\mathbf{r}_{2}$ are on the $x$ axis. $\lambda=500 \mathrm{~nm}$. Two materials are considered: lossy glass $\left(z_{0}=0.01 \lambda\right)$ and tungsten $\left(z_{0}=\right.$ $0.01 \lambda$ and $\left.z_{0}=0.1 \lambda\right)$. All curves are normalized by their maximum value at $\rho=0$. 
$z_{0}=0.01 \lambda$ (dotted curve). Thus, during the transition from the near to the far zone, the correlation length increases because evanescent waves vanish. The value $\lambda / 2$ of blackbody radiation is reached when $z \geq 0.1 \lambda$. Note that this increase in spatial coherence is not described by the far-field form of the van Cittert-Zernike theorem $[9,13]$, which describes an increase of spatial coherence due to propagation.

We now turn to the study of spatial coherence in light emission from materials supporting resonant surface waves, such as surface-plasmon or surface-phonon polaritons [14]. In the formalism used here, the existence of polaritons is taken into account in the Green dyadic $\overleftrightarrow{\mathbf{G}}$ which describes all the electrodynamic properties of the interface. In fact, it is well known that the $p$-polarized Fresnel transmission factor $t_{p}(\mathbf{K})$ appearing in Eq. (5) possesses a pole when $|\mathbf{K}|$ approaches the value $K_{s p}=k[\epsilon /(\epsilon+1)]^{1 / 2}$ of the wave vector of the polariton along the interface [14]. The thermal excitation of a surface polariton induces some spatial correlation in the field close to the surface, and we may expect a large increase of the correlation length.

We illustrate in Fig. 2 the effect of surface-plasmon [Fig. 2(a)] and surface-phonon [Fig. 2(b)] polaritons on the spatial coherence of the thermal near field. We plot in Fig. 2(a) the element $W_{x x}$ of the cross-spectral density tensor at the wavelength $\lambda=620 \mathrm{~nm}$, and in the plane $z_{0}=0.05 \lambda$, for three different metals. At this wavelength, their dielectric constants are $\epsilon=4.6+i 20.5$ for tungsten, $\epsilon=-8.26+i 1.12$ for gold, and $\epsilon=-15.04+i 1.02$ for silver. Both gold and silver exhibit surface-plasmon resonances at this wavelength. We clearly see that whereas the spatial correlation length for tungsten is a fraction of the wavelength (as in Fig. 1), the correlation length for gold and silver is much larger. In fact, although Fig. 2 is limited to $\rho<5 \lambda$ for the sake of visibility, the correlation extends on a distance given by the attenuation length of the surface-plasmon polariton. For gold and silver, the attenuation lengths are $16 \lambda$ and $65 \lambda$, respectively. The same effect is seen in Fig. 2(b) for a SiC crystal, which exhibits a surface-phonon polariton resonance at the wavelength $\lambda=11.36 \mu \mathrm{m}(\epsilon=-7.56+i 0.41)$ and no resonance at $\lambda=9.1 \mu \mathrm{m}(\epsilon=1.80+i 4.07)$. The emission of $\mathrm{SiC}$ gratings and the effect of surface-phonon waves were studied experimentally and numerically in Ref. [6]. The difference of behavior of this material at the two different wavelengths is striking in Fig. 2(b). The correlation length is much higher in the presence of the resonant surface wave (dashed line) than in the case where no surface wave is excited (solid line). The propagation distance of the surface-phonon polariton in this case is $36 \lambda$. In summary, we have shown how the delocalized electromagnetic surface mode, either coupled to a plasmon or a phonon in the material, correlates the near field on distances on the order of its propagation length, which easily reaches several tenths of wavelengths in the visible part of the spectrum. Physically, one could say that the collective resonance of the free electrons in the metals for plasmons, or of the crystal for phonons (lattice vibrations), transmits its spatial coherence to the electromagnetic field.

In order to demonstrate more precisely the role of the surface wave on the long-range correlation, we show in Fig. 3 the three diagonal elements $W_{k k}$ of the crossspectral density tensor, for gold, in the same conditions as in Fig. 2(a). It is well known that a surface polariton propagating along the $x$ axis is polarized in the $x-z$ plane [14]. Thus, when looking at the correlation between two points $\mathbf{r}_{1}$ and $\mathbf{r}_{2}$ that are along the $x$ axis, we expect to see a surface-wave induced correlation for the $x$ and $z$ components of the field only. This is exactly what is observed in Fig. 3. The elements $W_{x x}$ and $W_{z z}$ exhibit long-range correlation due to the surface wave, whereas $W_{y y}$ exhibits the same behavior as that of a metal without surface-wave resonance. This is a clear signature of the role of the surface polariton in increasing the spatial coherence. Although not shown here for brevity, the same result is obtained for $\mathrm{SiC}$ with surface-phonon excitation.

Another signature of the surface-wave excitation is the $z$ dependence of the electric energy density $u_{e}(z, \omega)=$ $\sum W_{k k}(\mathbf{r}, \mathbf{r}, \omega)$. Note that at aiven frequency $\omega, u_{e}$ is a function of $z$ only due to the translational invariance of the geometry in the $x-y$ plane. The energy density $u_{e}$, normalized by its far-field value, is plotted in Fig. 4 versus $z$. For materials without surface-wave resonances, such as glass and tungsten, the energy density increases at short distance $(z<0.1 \lambda)$. In fact, it is known that $u_{e}$ behaves like $1 / z^{3}$ in the very near field, due to nonradiating fields [8]. This result is retrieved in our calculation.

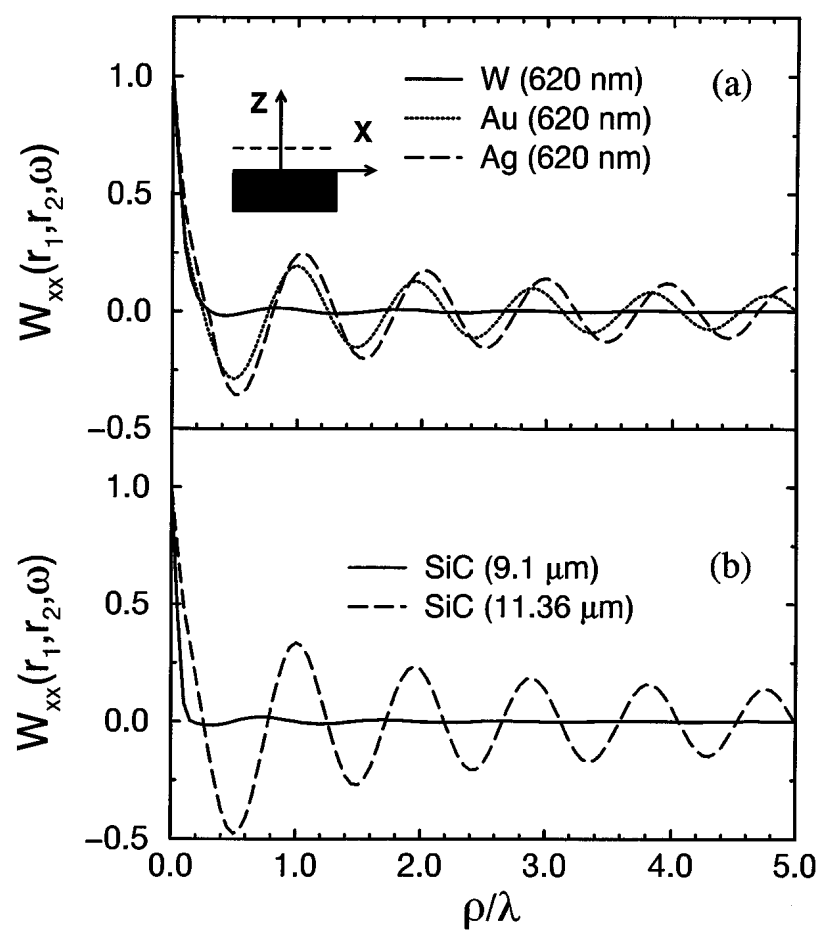

FIG. 2. Same as Fig. 1, with $z_{0}=0.05 \lambda$. (a) The case of three metals (tungsten, gold, and silver), $\lambda=620 \mathrm{~nm}$. (b) The case of $\mathrm{SiC}$ with $\lambda=9.1 \mu \mathrm{m}$ and $\lambda=11.36 \mu \mathrm{m}$. 


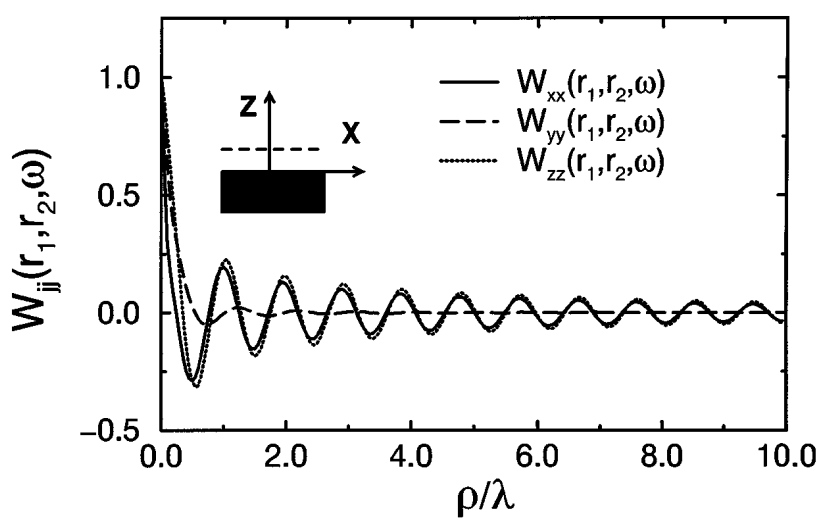

FIG. 3. Diagonal elements $W_{j j}\left(\mathbf{r}_{1}, \mathbf{r}_{2}, \omega\right)$ in the plane $z_{0}=$ $0.05 \lambda$ versus $\rho=\left|\mathbf{r}_{1}-\mathbf{r}_{2}\right|$, with $\mathbf{r}_{1}$ and $\mathbf{r}_{2}$ on the $x$ axis. Gold surface: $\lambda=620 \mathrm{~nm}$.

Also note that the existence of these nonradiating fields at short distances $z$ is responsible for the small peak observed at $\rho=0$ for glass in Fig. 1 (solid line). This is a striking difference between free blackbody radiation and the radiation produced at short distance by a blackbody radiator, which contains evanescent waves. The novel property is that for a material supporting a surface-plasmon polariton, such as gold in Fig. 4, the energy density starts to increase as soon as $z<\lambda$. This is so because the decay length of the surface wave in the $z$ direction is on the order of $\lambda$. The near-field enhancement is up to 100 times higher than that observed without surface-wave excitation. Although not shown here, we have observed the same effect with a surface-phonon polariton on a $\mathrm{SiC}$ surface. This behavior of the electric energy density shows that evanescent waves play a crucial role in radiative heat transfer at subwavelength scale when surface waves are excited.

We have shown that the thermal excitation of an electromagnetic surface mode dramatically changes the spatial correlation of the emitted field close to the surface. By using a grating as a coupler, the surface wave can be converted into propagating waves. This give rise to highly directional thermal emission at a given wavelength. This is a very unusual behavior for thermal sources. Since such a source emits light in particular directions and at particular frequencies, the spectrum of the emitted light strongly depends on the direction of observation. Thus, such a material is an example of a natural thermal source for which the field correlation in the source plane does not obey the scaling law established by Wolf [15]. Note that temporal and spatial coherence in thermal emission by silicon and $\mathrm{SiC}$ gratings has already been observed $[5,6]$. The analysis presented in this Letter explains the physical origin of this phenomenon.

To summarize, we have found novel correlation properties of fields produced by thermal sources in the near field: (i) Materials that do not support surface waves may display coherence lengths much shorter than $\lambda / 2$, (ii) ma-

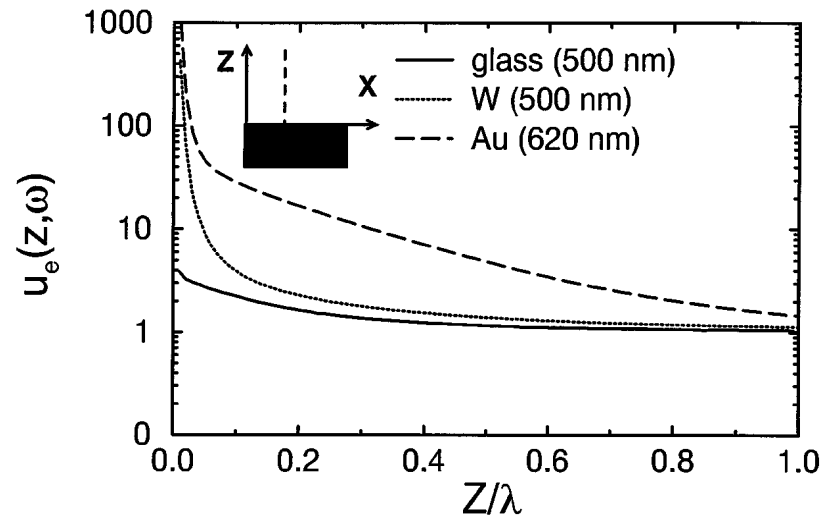

FIG. 4. Electric energy density $u_{e}(\mathbf{r}, \omega)$ versus $z$. The case of glass $(\lambda=500 \mathrm{~nm})$, tungsten $(\lambda=500 \mathrm{~nm})$, and gold $(\lambda=620 \mathrm{~nm})$. All curves are normalized by their far-field value at $z=10 \lambda$.

terials that support resonant surface waves exhibit longrange spatial correlations, (iii) the spectral electric density of energy is strongly enhanced close to an interface when a surface wave is excited, and (iv) an interface with a shallow grating supporting a surface wave is a natural thermal source whose spectrum depends on the emission direction.

[1] W.H. Carter and E. Wolf, J. Opt. Soc. Am. 65, 1067 (1975); E. Wolf, J. Opt. Soc. Am. 68, 6 (1978).

[2] Inverse Source Problems, edited by H. P. Baltes (SpringerVerlag, Berlin, 1978), p. 123.

[3] C. L. Tien and G. Chen, J. Heat Transfer 114, 799 (1994).

[4] C. C. Williams and H. K. Wickramasinghe, Appl. Phys. Lett. 49, 1587 (1986).

[5] P. J. Hesketh et al., Nature (London) 324, 549 (1986); Phys. Rev. B 37, 10803 (1988).

[6] J. Le Gall et al., Phys. Rev. B 55, 10105 (1997).

[7] L. D. Landau, E. M. Lifshitz, and L.P. Pitaevskii, Statistical Physics (Pergamon Press, Oxford, 1980), 3rd ed., Part 1, Chap. XII and Part 2, Chap. VIII.

[8] S. M. Rytov, Yu. A. Kravtsov, and V.I. Tatarskii, Principles of Statistical Radiophysics (Springer-Verlag, Berlin, 1989), Vol. 3, Chap. 3.

[9] L. Mandel and E. Wolf, Optical Coherence and Quantum Optics (Cambridge University Press, Cambridge, England, 1995).

[10] D. C. Bertilone, J. Mod. Opt. 43, 207 (1996); J. Opt. Soc. Am. A 14, 693 (1997).

[11] J. E. Sipe, J. Opt. Soc. Am. B 4, 481 (1987).

[12] R. Carminati et al., in Heat Transfer 1998, edited by J. S. Lee, Proceedings of the 11th International Heat Transfer Conference (Korean Society of Mechanical Engineering, Kyongju, 1998), Vol. 7, p. 427.

[13] J.W. Goodman, Statistical Optics (Wiley, New York, 1985), p. 206.

[14] Surface Polaritons, edited by V. M. Agranovich and D. L. Mills (North-Holland, Amsterdam, 1982).

[15] E. Wolf, Phys. Rev. Lett. 56, 1370 (1986). 